University of New Orleans

ScholarWorks@UNO

$12-1978$

\title{
Propagation of partially polarized light through anisotropic media with or without depolarization: A differential $4 \times 4$ matrix calculus
}

R. M.A. Azzam

University of New Orleans, razzam@uno.edu

Follow this and additional works at: https://scholarworks.uno.edu/ee_facpubs

Part of the Electrical and Electronics Commons, and the Physics Commons

\section{Recommended Citation}

R. M. A. Azzam, "Propagation of partially polarized light through anisotropic media with or without depolarization: A differential $4 \times 4$ matrix calculus," J. Opt. Soc. Am. 68, 1756-1767 (1978)

This Article is brought to you for free and open access by the Department of Electrical Engineering at ScholarWorks@UNO. It has been accepted for inclusion in Electrical Engineering Faculty Publications by an authorized administrator of ScholarWorks@UNO. For more information, please contact scholarworks@uno.edu. 


\section{CONCLUSION}

The application of the proportional medium theory to radial media produces a wealth of subtle unexpected phenomena, which run from the possibility of "leaky modes," even for zero partial wave number, to a splitting of the guided modes, that is found to be stable with increasing frequency, and to the existence of modes for which the effective radial medium is entirely negative! If we consider the unusual features of the proportional medium equation, these results may not be so surprising. In any case, it would be very hard to pursue this research with confidence if it were not possible to ballast it with two main guidelines: (1) the proportional medium equation is exact for a conceivable medium. (2) The conformity of the behavior of all the solutions at the reflection curves can be used as a check on any further mathematical approximation put forward.

The uniform approximation derived in Sec. V is found to dive through the singularity in exactly the right way. It follows that for high frequencies at least the qualitative features, of the theory here enumerated, are correct. Nonetheless the results obtained are sufficiently important to warrant further careful verification, based on direct numerical integration of the proportional medium equation or even of the coupled equations from which it is derived.

*Research sponsored by CNPq, FINEP, and BNDE.
${ }^{1}$ M. Matsuhara, "Analysis of electromagnetic-wave modes in lens-like media," J. Opt. Soc. Am. 63, 135 (1973).

${ }^{2} \mathrm{R}$. Yamada and $Y$. Inabe, "Guided waves in an optical square law medium," J. Opt. Soc. Am. 64, 964 (1964).

${ }^{3} \mathrm{C}$. N. Kurtz and W. Streifer, "Guided waves in inhomogeneous focussing media. Part I: Formulation for quadratic inhomogeneity," IEEE Trans. Microwave Theory Tech. 17, 11 (1969). ${ }^{4}$ C. N. Kurtz and W. Streifer, "Guided waves in inhomogeneous focussing media. Part II: Asymptotic solution for general weak inhomogeneity," IEEE Trans. Microwave Theory Tech. 17, 250 (1969).

${ }^{5} \mathrm{D}$. Marcuse, Light Transmission Optics (Van Nostrand, New York, 1972).

${ }^{6} \mathrm{~B}$. W. Batterman and H. Cole, "Dynamical diffraction of $\mathrm{x}$ rays by perfect crystals," Rev. Mod. Phys. 36, 681 (1964).

${ }^{7}$ I. G. Petrovsky, Partial Differential Equations (Interscience, New York, 1954), p. 26.

${ }^{8} \mathrm{M}$. Born and E. Wolf, Principles of Optics, 4th ed. (Pergamon, Oxford, 1970), p. 32.

${ }^{9} \mathrm{~J}$. R. Wait, Electromagnetic Waves in Stratified Media (Pergamon, Oxford, 1970), p. 66 and 80.

${ }^{10} \mathrm{E}$. L. Ince, Ordinary Differential Equations (Dover, New York, 1956), p. 396.

${ }^{11} \mathrm{R}$. E. Langer, "On the connection formulas and solutions of the wave equation," Phys. Rev. 51, 669-76 (1937).

${ }^{12}$ M. V. Berry and A. M. Ozorio de Almeida, "Semiclassical Approximation of the radial equation with two-dimensional potentials," J. Phys. A 6, 1451-1460 (1973).

${ }^{13}$ S. C. Miller and R. H. Good, "A WKB-type approximation to the Schrödinger equation," Phys. Rev. 91, 174-9 (1953).

${ }^{14} \mathrm{H}$. Abramowitz and I. Stegun, Handbook of Mathematical Functions, Natl. Bur. Stand. (U.S. GPO, Washington, 1964), Chap. 10.

\title{
Propagation of partially polarized light through anisotropic media with or without depolarization: A differential $4 \times 4$ matrix calculus
}

\author{
R. M. A. Azzam \\ Hematology Division and Department of Internal Medicine, University of Nebraska Medical Center, Omaha, Nebraska 68105 \\ and Electrical Materials Laboratory, College of Engineering, University of Nebraska, Lincoln, Nebraska 68588 \\ (Received 4 April 1977)

\begin{abstract}
We extend the scope of the Mueller calculus to parallel that established by Jones for his calculus. We find that the Stokes vector $\mathbf{S}$ of a light beam that propagates through a linear depolarizing anisotropic medium obeys the first-order linear differential equation $d S / d z=\mathrm{mS}$, where $z$ is the distance traveled along the direction of propagation and $\mathrm{m}$ is a $4 \times 4$ real matrix that summarizes the optical properties of the medium which influence the Stokes vector. We determine the differential matrix $m$ for eight basic types of optical behavior, find its form for the most general anisotropic nondepolarizing medium, and determine its relationship to the complex $2 \times 2$ differential Jones matrix. We solve the Stokes-vector differential equation for light propagation in homogeneous nondepolarizating media with arbitrary absorptive and refractive anisotropy. In the process, we solve the differential-matrix and Mueller-matrix eigenvalue equations. To illustrate the case of inhomogeneous anisotropic media, we consider the propagation of partially polarized light along the helical axis of a cholesteric or twisted-nematic liquid crystal. As an example of depolarizing media, we consider light propagation through a medium that tends to equalize the preference of the state of polarization to the right and left circular states.
\end{abstract}

\section{INTRODUCTION}

The state of polarization of a light beam changes continuously as it propagates through an optically anisotropic medium. When the light beam is totally polarized and the medium is linear and nondepolarizing, the evolution of the state of polarization along the direction of propa- gation can be described by the simple linear first-order differential equation ${ }^{1} d \mathrm{E} / d z=\mathrm{NE}$, where $\mathbf{E}$ is the $2 \times 1$. Jones vector of the beam, $z$ is the distance traveled along the direction of propagation, and $\mathrm{N}$ is a $2 \times 2$ complex matrix that describes the optical properties of the medium. ${ }^{2}$ However, this description becomes inapplicable when the beam is partially polarized or when the 
medium is depolarizing. We deal in this paper with this more general case. We find in Sec. II that for partially polarized light propagating in a linear medium that may exhibit depolarization, the state of polarization is governed by another linear first-order differential equation $d \mathrm{~S} / d z=\mathrm{mS}$, where $\mathrm{S}$ is the $4 \times 1$ Stokes vector of the beam $^{3}$ (whose elements are the four Stokes parameters $S_{0}, S_{1}, S_{2}$, and $\left.S_{3}\right), z$ is the distance traveled along the direction of propagation, and $\mathrm{m}$ is a $4 \times 4$ real matrix that summarizes the optical properties of the medium. In Sec. III we determine the matrix $m$ for different anisotropic media and also find its relationship to the $2 \times 2$ Jones matrix N. In Sec. IV we solve the Stokes-vector differential equation for homogeneous nondepolarizing media with arbitrary absorptive and refractive anisotropy. In Sec. V we deal with the case of inhomogeneous anisotropic media and take the classic problem of light propagation along the helical axis of a cholesteric or twisted-nematic liquid crystal as an example. The propagation of light through depolarizing media is illustrated in Sec. VI by a simple example. Section VII contains some concluding remarks on this paper.

\section{THE STOKES-VECTOR DIFFERENTIAL EQUATION}

Consider the propagation of a light beam along the $z$ axis of an $x y z$ Cartesian right-handed coordinate system in a linear optically anisotropic medium that may exhibit depolarization (Fig. 1). We assume that the medium is transversally homogeneous (along the $x$ and $y$ axes), but that it is possibly longitudinally inhomogeneous (along the $z$ axis). We characterize the beam by its Stokes vector $\mathrm{S}(z)$ which varies along the direction of propagation $z$ because of the anisotropic and depolarization properties of the medium. The Stokes vectors at $z$ and $z$ $+\Delta z$ are interrelated by

$$
\mathrm{S}(z+\Delta z)=\mathbf{M}_{z, \Delta z} \mathbf{S}(z),
$$

where $\mathbf{M}_{z, \Delta z}$ is the Mueller matrix of a thin slab of the medium located at the coordinate $z$ and of incremental thickness $\Delta z$ (the slab bounded at $z$ and $z+\Delta z$ by two planes perpendicular to the $z$ axis). We subtract $\mathrm{S}(z)$ from both sides of Eq. (1):

$$
\mathrm{S}(z+\Delta z)-\mathrm{S}(z)=\left(\mathrm{M}_{z, \Delta z}-\mathrm{I}\right) \mathrm{S}(z),
$$

where $I$ is the $4 \times 4$ identity matrix. If we divide both sides of Eq. (2) by $\Delta z$ and take the limit as $\Delta z \rightarrow 0$, we obtain $^{4}$

$$
d \mathbf{S} / d z=\mathrm{mS},
$$

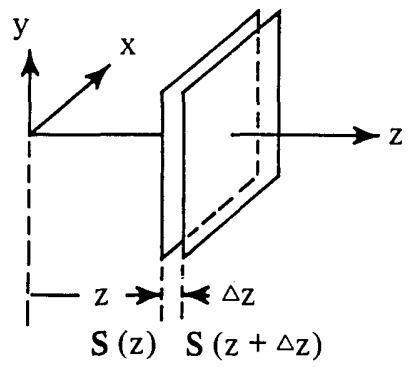

FIG. 1. Partially polarized light is assumed to propagate along the $z$ axis of an $x y z$ Cartesian coordinate system in an anisotropic medium that may exhibit depolarization. $\mathrm{S}(z)$ and $\mathrm{S}(z+\Delta z)$ are the Stokes vectors of the beam at two transverse planes located at $z$ and $z+\Delta z$, respectively. where

$$
\mathrm{m}=\lim _{\Delta z-0} \frac{\mathrm{M}_{z, \Delta z}-\mathrm{I}}{\Delta z} .
$$

Equation (3) is the desired first-order linear differential equation in the Stokes vector $S$. The $4 \times 4$ matrix $\mathrm{m}$ that appears in Eq. (3) and which is defined by Eq. (4) summarizes the optical properties of the medium that influence the intensity and state of polarization of the beam. $m$ may be called the differential propagation matrix of the medium in the Stokes vector-Mueller matrix formulation, but we will simply refer to it as the differential matrix in this paper.

Equation (3) is equivalent to four simultaneous firstorder linear differential equations in the four Stokes parameters $S_{0}, S_{1}, S_{2}$, and $S_{3}$. Many of the mathematical tools presently available for the solution of such a set of equations can be brought to bear on the problem of propagation of partially polarized light through anisotropic media. This will be illustrated in Secs. IV and V by specific examples. In general, the solution of Eq.

(3) gives a Stokes vector that is a function of $z$ :

$$
\mathbf{S}(z)=\left[S_{0}(z), S_{1}(z), S_{2}(z), S_{3}(z)\right] .
$$

The first Stokes parameter $S_{0}(z)$ describes the total intensity of the light beam as a function of the distance $z$ traveled in the medium. If the remaining three Stokes parameters are divided by $S_{0}(z)$, the resulting normalized parameters

$$
\begin{aligned}
& s_{1}(z)=S_{1}(z) / S_{0}(z), \\
& s_{2}(z)=S_{2}(z) / S_{0}(z), \\
& s_{3}(z)=S_{3}(z) / S_{0}(z),
\end{aligned}
$$

can be considered as the components of a three-dimensional vector

$$
\mathbf{s}=\left[s_{1}, s_{2}, s_{3}\right]
$$

that specifies the state of polarization of the beam completely. ${ }^{3}$ The length of the vector $\mathbf{s}$,

$$
|\mathbf{s}|=\left(s_{1}^{2}+s_{2}^{2}+s_{3}^{2}\right)^{1 / 2} \text {, }
$$

gives the degree of polarization of the beam. The angle between the vector $\mathbf{s}$ and the $s_{3}$ axis, of an $s_{1} s_{2} s_{3}$ Cartesian coordinate system, is $\left(\frac{1}{2} \pi-2 \epsilon\right)$, while the angle between the plane of $\mathbf{s}$ and the $s_{3}$ axis and the $s_{1} s_{3}$ coordinate plane is $2 \theta$, where $\theta$ and $\epsilon$ are the azimuth and ellipticity angle of the polarization ellipse of the totally polarized component of the beam (Fig. 2). The locus of the end point of $\mathrm{s}$ is a three-dimensional curve $\mathrm{C}$ that provides an interesting graphical representation of the evolution of the state of polarization of the beam as it propagates through the anisotropic medium. Such a curve has to be confined inside or on the surface of the sphere of unit radius centered at the origin, the Poincaré sphere, because the degree of polarization of the beam cannot exceed one. Notice that if the vector $\mathbf{s}$ is radially extended to intersect the unit sphere, the locus of the point of intersection gives the evolution of the polarization ellipse of the totally polarized component of the beam in the familiar Poincaré-sphere representation. ${ }^{3}$ A subsequent stereographic projection onto a 


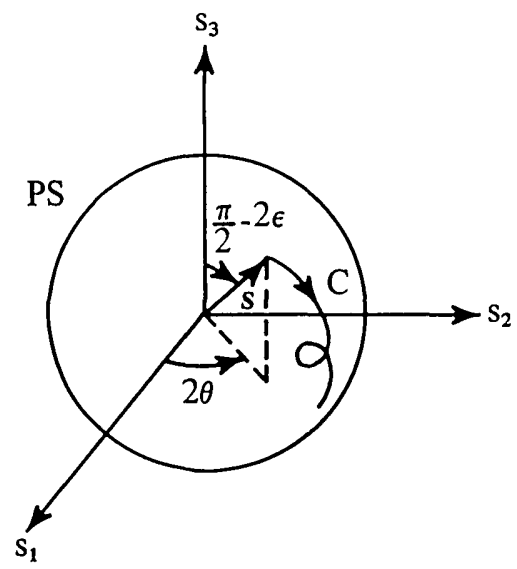

FIG. 2. The normalized Stokes parameters $s_{1}, s_{2}, s_{3}$

[Eqs. (6a)] constitute the components of a vector $\mathbf{s}$ that represents the state of polarization of partially polarized light completely. $|\mathbf{B}|$ gives the degree of polarization of the beam while the angular spherical coordinates $\left(\frac{1}{2} \pi-2 \epsilon\right)$ and $2 \theta$ determine the ellipticity angle $\epsilon$ and azimuth $\theta$ of the polarization ellipse of the totally polarized component of the beam. The trajectory $\mathrm{C}$ of the endpoint of $\mathbf{B}$ gives an interesting graphical representation of the evolution of the state of polarization as light propagates through an anisotropic medium. The unit sphere in the $s_{1} s_{2} s_{3}$ space is the Poincaré sphere (PS).

plane tangent to the sphere gives still another representation of the ellipse of polarization. The latter can be considered as a generalization of the complex-plane trajectorites that describe the propagation of totally polarized light in anisotropic nondepolarizing media discussed elsewhere. ${ }^{2,5}$

\section{THE DIFFERENTIAL MATRIX m}

\section{A. General properties}

With reference to $F$ ig. 1 , let $M_{z}$ be the Mueller matrix of the slab of the medium bounded by the $x y$ plane and the plane parallel to it a distance $z$ away along the direction of propagation. The Stokes vector $\mathrm{S}(z)$ at $z$ is equal to $\mathrm{M}_{z}$ times the initial Stokes vector $\mathrm{S}(0)$ at $z=0$ :

$$
\mathrm{S}(z)=\mathrm{M}_{z} \mathrm{~S}(0) \text {. }
$$

Equation (8) is the form that the general solution of Eq. (3) must take subject to the initial condition $S=S(0)$ at $z=0$, from which it is concluded that $\mathrm{M}_{0}=\mathrm{I}$ identically. By differentiation of $\mathrm{Eq}$. (8) we get

$$
d \mathbf{S}(z) / d z=\left(d \mathbf{M}_{z} / d z\right) \mathbf{S}(0) \text {. }
$$

Subsequent substitution of $d \mathbf{S} / d z$ from Eq. (3) into Eq. (9) gives

$$
\operatorname{mS}(z)=\left(d \mathbf{M}_{z} / d z\right) \mathbf{S}(0),
$$

or

$$
\mathrm{mM}_{z} \mathrm{~S}(0)=\left(d \mathrm{M}_{z} / d z\right) \mathbf{S}(0),
$$

if Eq. (8) is used. Finally if we drop $\mathbf{S}(0)$ from $\mathrm{Eq}$. (10), we obtain

$$
\begin{aligned}
& d \mathrm{M}_{z} / d z=\mathrm{mM}_{z}, \\
& \mathrm{~m}=\left(d \mathrm{M}_{z} / d z\right) \mathrm{M}_{z}^{-1} .
\end{aligned}
$$

Equations (11) and (12) relate the differential matrix m, describing the intensive properties of (a thin slab of) the medium, to the Mueller matrix $\mathrm{M}_{z}$, describing the extensive properties of (a thick slab of) the same medium. ${ }^{6}$ Equation (12) may be considered as an alternative definition of $\mathrm{m}$.

If we post- and premultiply both sides of Eq. (4) by the $4 \times 4$ rotation and counter-rotation (Mueller) matrices $R(\theta)$ and $R(-\theta)$, respectively, enter these matrices within the field of the limit sign, and use the associative law of matrix algebra and the identity $R(\theta) R(-\theta)=I$, we obtain

$\mathrm{R}(-\theta) \mathrm{mR}(\theta)=\lim _{\Delta z \rightarrow 0} \frac{\mathrm{R}(-\theta) \mathrm{M}_{z, \Delta z} \mathrm{R}(\theta)-\mathrm{I}}{\Delta z}$,

or

$$
\mathrm{m}^{\prime}=\mathrm{R}(-\theta) \mathrm{mR}(\theta),
$$

where

$$
\begin{aligned}
& \mathrm{m}^{\prime}=\lim _{\Delta z \rightarrow 0} \frac{\mathrm{M}_{z, \Delta z}^{\prime}-\mathrm{I}}{\Delta z}, \\
& \mathrm{M}_{z, \Delta z}^{\prime}=\mathrm{R}(-\theta) \mathrm{M}_{z, \Delta z} \mathrm{R}(\theta),
\end{aligned}
$$

and $^{3}$

$$
R(\theta)=\left(\begin{array}{cccc}
1 & 0 & 0 & 0 \\
0 & \cos 2 \theta & \sin 2 \theta & 0 \\
0 & -\sin 2 \theta & \cos 2 \theta & 0 \\
0 & 0 & 0 & 1
\end{array}\right) .
$$

$\mathrm{m}^{\prime}$ of Eq. (15) represents the differential matrix of the medium in a new coordinate system $x^{\prime} y^{\prime} z$ which is rotated with respect to the old coordinate system $x y z$ by the angle $\theta$ around the $z$ axis. Notice that the transformation of the differential matrix $\mathrm{m}$, from $\mathrm{m}$ to $\mathrm{m}^{\prime}$, due to a coordinate rotation obeys the same law, Eq. (14), as that which governs the transformation of the Mueller matrix, ${ }^{6} \mathrm{Eq}$. (16).

\section{B. The differential matrix $\mathrm{m}$ for different} nondepolarizing anisotropic media

We now determine the differential matrix $\mathrm{m}$ for different nondepolarizing optically anisotropic (and isotropic) media. Specifically, we consider the eight basic types of optical behavior into which an arbitrary optical behavior can be resolved, according to the lamellar representation suggested by Jones. ${ }^{1}$ The eight optical properties are $(1,2)$ isotropic refraction and absorption, $(3,4)$ linear birefringence and linear dichroism along the $x y$ coordinate axes, $(5,6)$ linear birefringence and linear dichroism along the bisectors of the $x y$ coordinate axes, and $(7,8)$ circular birefringence and circular dichroism.

Equation (4) easily provides the differential matrix $\mathrm{m}$ from the Mueller matrix of a thin slab of the given medium. As an example, consider the case of a linearly birefringent medium with principal (fast and slow) axes aligned with the $x$ and $y$ axes of the reference coordinate system. The Mueller matrix of a slab of arbitrary thickness $z$ is given by ${ }^{3}$ 
TABLE I. The differential $4 \times 4$ matrix m and Jones $2 \times 2$ matrix $\mathbf{N}$ for eight basic types of optical behavior. ${ }^{\mathbf{a}}$

Optical property

$\mathrm{m}$

$\mathrm{N}$

1. Isotropic refraction

$$
\left(\begin{array}{llll}
0 & 0 & 0 & 0 \\
0 & 0 & 0 & 0 \\
0 & 0 & 0 & 0 \\
0 & 0 & 0 & 0
\end{array}\right)
$$$$
\frac{\varphi}{2}\left(\begin{array}{ll}
i & 0 \\
0 & i
\end{array}\right)
$$

2. Isotropic absorption

$$
\left(\begin{array}{cccc}
\alpha & 0 & 0 & 0 \\
0 & \alpha & 0 & 0 \\
0 & 0 & \alpha & 0 \\
0 & 0 & 0 & \alpha
\end{array}\right) \quad \frac{\alpha}{2}\left(\begin{array}{cc}
1 & 0 \\
0 & 1
\end{array}\right)
$$

3. Linear birefringence along the $x y$ coordinate axes

$$
\left(\begin{array}{rrrr}
0 & 0 & 0 & 0 \\
0 & 0 & 0 & 0 \\
0 & 0 & 0 & \eta \\
0 & 0 & -\eta & 0
\end{array}\right)
$$

$$
\frac{\eta}{2}\left(\begin{array}{rr}
i & 0 \\
0 & -i
\end{array}\right)
$$

4. Linear dichroism along the $x y$ coordinate axes

$$
\left(\begin{array}{llll}
0 & \beta & 0 & 0 \\
\beta & 0 & 0 & 0 \\
0 & 0 & 0 & 0 \\
0 & 0 & 0 & 0
\end{array}\right)
$$$$
\frac{\beta}{2}\left(\begin{array}{rr}
1 & 0 \\
0 & -1
\end{array}\right)
$$

5. Linear birefringence along the bisectors of the $x y$ coordinate axes

$$
\left(\begin{array}{rrrr}
0 & 0 & 0 & 0 \\
0 & 0 & 0 & \nu \\
0 & 0 & 0 & 0 \\
0 & -\nu & 0 & 0
\end{array}\right) \quad-\frac{\nu}{2}\left(\begin{array}{ll}
0 & i \\
i & 0
\end{array}\right)
$$

6. Linear dichroism along the bisectors of the $x y$ coordinate axes

$$
\left(\begin{array}{llll}
0 & 0 & \gamma & 0 \\
0 & 0 & 0 & 0 \\
\gamma & 0 & 0 & 0 \\
0 & 0 & 0 & 0
\end{array}\right)
$$$$
\frac{\gamma}{2}\left(\begin{array}{ll}
0 & 1 \\
1 & 0
\end{array}\right)
$$

7. Circular birefringence

$$
\left(\begin{array}{rrrr}
0 & 0 & 0 & 0 \\
0 & 0 & \mu & 0 \\
0 & -\mu & 0 & 0 \\
0 & 0 & 0 & 0
\end{array}\right) \quad-\frac{\mu}{2}\left(\begin{array}{rr}
0 & -1 \\
1 & 0
\end{array}\right)
$$

8. Circular dichroism

$$
\left(\begin{array}{rrrr}
0 & 0 & 0 & \delta \\
0 & 0 & 0 & 0 \\
0 & 0 & 0 & 0 \\
\delta & 0 & 0 & 0
\end{array}\right) \quad \frac{\delta}{2}\left(\begin{array}{rr}
0 & -i \\
i & 0
\end{array}\right)
$$

${ }^{\text {a }}$ The notation used in this table is different from that of Jones in Ref. 1. $\phi, \alpha$ equal double the propagation constant and double the amplitude extinction coefficient, respectively, of the isotropic medium. $\eta, \beta$ represent, respectively, the difference between the principal propagation constants and absorption coefficients for the $x$ and $y$ linear eigenpolarizations with similar meanings for $\nu, \gamma$, and $\mu, \delta$.

$$
\mathbf{M}_{z}=\left(\begin{array}{cccc}
1 & 0 & 0 & 0 \\
0 & 1 & 0 & 0 \\
0 & 0 & \cos \eta z & \sin \eta z \\
0 & 0 & -\sin \eta z & \cos \eta z
\end{array}\right),
$$

where

$$
\eta=4 \pi \Delta n / \lambda \text {. }
$$

$\Delta n$ is the birefringence of the medium (i. e., the difference between the principal $x$ and $y$ refractive indices) and $\lambda$ is the wavelength of light. For a thin slab of incremental thickness $\Delta z$, the Mueller matrix $\mathrm{M}_{\Delta z}$ is obtained by expanding the cosine and sine functions in Eq. (18) into their power series and retaining only the firstorder terms in $\Delta z$; this gives

$$
\mathbf{M}_{\Delta z}=\left(\begin{array}{cccc}
1 & 0 & 0 & 0 \\
0 & 1 & 0 & 0 \\
0 & 0 & 1 & \eta \Delta z \\
0 & 0 & -\eta \Delta z & 1
\end{array}\right) .
$$

Direct substitution of Eq. (20) into Eq. (4) yields the following differential matrix for the linearly birefringent medium:

$$
\mathrm{m}=\left(\begin{array}{cccc}
0 & 0 & 0 & 0 \\
0 & 0 & 0 & 0 \\
0 & 0 & 0 & \eta \\
0 & 0 & -\eta & 0
\end{array}\right)
$$

The same result could also have been obtained easily by substituting Eq. (18) into Eq. (12) noting that $\mathbf{M}_{z}^{-1}$ and $d \mathbf{M}_{z} / d z$ are readily determined from $\mathbf{M}_{z}$ in this case. ${ }^{7}$

We applied the same procedure as discussed above for linear birefringence along the coordinate axes to determine $\mathrm{m}$ for nondepolarizing media that exhibit the seven other fundamental optical properties listed earlier. The results are summarized in Table I, which also lists the corresponding $2 \times 2$ complex differential Jones matrices, for reference and comparison.

It is interesting to note that the differential matrix $\mathrm{m}$ for an optically isotropic transparent medium is the null matrix. This is expected because the information on the absolute phase of the wave (the only wave property that would change in an isotropic transparent medium) is suppressed in the definition of the Stokes parameters. This makes the Mueller matrix of a slab of any thickness of such a medium equal to the identity matrix and Eqs. (4) and (12) both yield $m=0$. It follows that, in the present $4 \times 4$ matrix formulation, an arbitrary anisotropy (without depolarization) is actually resolvable into seven (instead of eight) basic properties if the same lamellar representation as that suggested by Jones ${ }^{1}$ is used.

\section{General relationship between the differential $4 \times 4$ matrix $\mathrm{m}$ and Jones differential $2 \times 2$ matrix $\mathrm{N}$}

We now determine the general relationship between the (intensive) differential $4 \times 4$ real matrix $\mathrm{m}$ and Jones differential $2 \times 2$ complex matrix $N$. We make use of the already established relationship between the (extensive) Mueller and Jones matrices. Specifically, if the Jones matrix of an arbitrarily thick slab of the medium is denoted by 


$$
\mathrm{J}=\left(\begin{array}{ll}
J_{1} & J_{3} \\
J_{4} & J_{2}
\end{array}\right)
$$

the corresponding Mueller matrix of the same slab is given by ${ }^{3}$

$$
\mathrm{M}=\left(\begin{array}{cccc}
\left(E_{1}+E_{2}+E_{3}+E_{4}\right) / 2 & \left(E_{1}-E_{2}-E_{3}+E_{4}\right) / 2 & F_{13}+F_{42} & -D_{13}-D_{42} \\
\left(E_{1}-E_{2}+E_{3}-E_{4}\right) / 2 & \left(E_{1}+E_{2}-E_{3}-E_{4}\right) / 2 & F_{13}-F_{42} & -D_{13}+D_{42} \\
F_{14}+F_{32} & F_{14}-F_{32} & F_{12}+F_{34} & -D_{12}+D_{34} \\
D_{14}+D_{32} & D_{14}-D_{32} & D_{12}+D_{34} & F_{12}-F_{34}
\end{array}\right)
$$

where

$$
E_{i}=\left|J_{i}\right|^{2}, \quad F_{i k}=\operatorname{Re}\left(J_{i} J_{k}^{*}\right), \quad D_{i k}=\operatorname{Im}\left(J_{i}^{*} J_{k}\right), \quad i, k=1, \ldots, 4 .
$$

The Jones matrix $\mathrm{J}$ of a thin slab of incremental thickness $\Delta z$ of a medium with a differential matrix $\mathrm{N}$ is given by

$$
\mathrm{J}=\mathrm{I}+\mathrm{N} \Delta z
$$

from the definition of $\mathrm{N}^{1}$ where $\mathrm{I}$ is the $2 \times 2$ identity matrix. If we substitute

$$
\mathbf{N}=\left(\begin{array}{ll}
n_{1} & n_{3} \\
n_{4} & n_{2}
\end{array}\right), \quad n_{k}=n_{k}^{r}+i n_{k}^{i}, \quad k=1, \ldots, 4
$$

into Eq. (25) we obtain

$$
J=\left(\begin{array}{cc}
1+n_{1} \Delta z & n_{3} \Delta z \\
n_{4} \Delta z & 1+n_{2} \Delta z
\end{array}\right)
$$

To obtain the Mueller matrix of the same thin slab, we apply to the Jones matrix of Eq. (27) the transformation specified by Eqs. (22)-(24); this yields a matrix that can be put in the form

$$
\mathrm{M}=\mathrm{I}+\mathrm{m} \Delta z
$$

to first order in $\Delta z$, where I is the $4 \times 4$ identity matrix and

$\mathrm{m}=\left(\begin{array}{cccc}n_{1}^{r}+n_{2}^{r} & n_{1}^{r}-n_{2}^{r} & n_{3}^{r}+n_{4}^{r} & -n_{3}^{i}+n_{4}^{i} \\ n_{1}^{r}-n_{2}^{r} & n_{1}^{r}+n_{2}^{r} & n_{3}^{r}-n_{4}^{r} & -n_{3}^{i}-n_{4}^{i} \\ n_{3}^{r}+n_{4}^{r} & -n_{3}^{r}+n_{4}^{r} & n_{1}^{r}+n_{2}^{r} & n_{1}^{i}-n_{2}^{i} \\ -n_{3}^{i}+n_{4}^{i} & n_{3}^{i}+n_{4}^{i} & -n_{1}^{i}+n_{2}^{i} & n_{1}^{r}+n_{2}^{r}\end{array}\right)$

m of Eq. (29) is the desired differential $4 \times 4$ matrix of the anisotropic nondepolarizing medium whose $2 \times 2$ differential Jones matrix is $\mathrm{N}$ of Eq. (26). If we construct the sum $(+)$ and difference $(-)$ of the diagonal $(d)$ and offdiagonal $(f)$ elements of the $\mathrm{N}$ matrix

$$
\begin{array}{ll}
d_{+}=n_{1}+n_{2}, & d_{-}=n_{1}-n_{2}, \\
f_{+}=n_{3}+n_{4}, & f_{-}=n_{3}-n_{4},
\end{array}
$$

we can readily rewrite Eq. (29) as

$$
\mathrm{II}=\left(\begin{array}{cccc}
d_{+}^{r} & d_{-}^{r} & f_{+}^{r} & -f_{-}^{i} \\
d_{-}^{r} & d_{+}^{r} & f_{-}^{r} & -f_{+}^{i} \\
f_{+}^{r} & -f_{-}^{r} & d_{+}^{r} & d_{-}^{i} \\
-f_{-}^{i} & f_{+}^{i} & -d_{-}^{i} & d_{+}^{r}
\end{array}\right) .
$$

Equation (31) shows that the elements of $\mathrm{m}$ are simply the real and imaginary parts (indicated by the superscripts $r$ and $i$, respectively) of the sum of and difference of the diagonal and off-diagonal elements of $\mathrm{N}$. Furthermore, if $\mathrm{m}$ is partitioned into four $2 \times 2$ submatrices,

$$
\mathrm{m}=\left(\begin{array}{ll}
\mathrm{m}_{1} & \mathrm{~m}_{3} \\
\mathrm{~m}_{4} & \mathrm{~m}_{2}
\end{array}\right)
$$

we find that the diagonal submatrices $m_{1}$ and $m_{2}$ of $m$ are determined only by the diagonal elements $n_{1}$ and $n_{2}$ of $\mathrm{N}$ and, likewise, the off-diagonal submatrices $m_{3}$ and $m_{4}$ of $m$ are determined only by the off-diagonal elements $n_{3}$ and $n_{4}$ of $\mathrm{N}$.

Equation (31) also shows that the differential matrix $\mathrm{m}$ of any anisotropic nondepolarizing medium is determined by seven independent parameters only, namely the real and imaginary parts of $d_{-}, f_{+}$, and $f_{\rightarrow}$, and the real part of $d_{+^{*}}$ (The imaginary part of $d_{+}, d_{+}^{i}$, does not appear. ${ }^{8}$ )

The reader can easily verify that the $\mathrm{m}$ and $\mathrm{N}$ matrices of the eight types of optical behavior listed in Table I do indeed satisfy the general relationship between $\mathrm{m}$ and $\mathrm{N}$ of Eqs. (26) and (29).

According to Jones lamellar representation, ${ }^{1}$ a thin slab of a given medium is equivalent to a stack of eight subslabs all of equal thickness ( $\frac{1}{8}$ th of that of the original slab) and each possessing one and only one of the eight fundamental properties listed in Table I. Furthermore, the $\mathrm{N}$ matrix of the medium of the thin slab is the sum of the $\mathrm{N}$ matrices of the media of the subslabs. The same lamellar representation is valid in the present formulation. Consequently, the most general $\mathrm{m}$ matrix of a nondepolarizing medium can also be obtained by adding the $m$ matrices that correspond to the different optical properties listed in Table $\mathrm{I}^{9}$ :

$$
\mathrm{m}=\left(\begin{array}{cccc}
\alpha & \beta & \gamma & \delta \\
\beta & \alpha & \mu & \nu \\
\gamma & -\mu & \alpha & \eta \\
\delta & -\nu & -\eta & \alpha
\end{array}\right)
$$

It is significant to observe that the fundamental optical properties given in Table I contribute to $\mathrm{m}$ elements in 
mutually exclusive matrix positions. Specifically, we note that (1) elements along the leading diagonal which are all equal $\left(m_{k k}=\alpha, k=1, \ldots, 4\right)$ represent isotropic absorption, (2) elem ents along the secondary diagonal represent circular birefringence $\left(m_{23}=-m_{32}=\mu\right)$ and circular dichroism $\left(m_{14}=m_{41}=\delta\right)$, and (3) the remaining elements in the first row and first column represent linear dichroism along the coordinate axes $\left(m_{12}=m_{21}\right.$ $=\beta$ ) and linear dichroism along the bisectors of the coordinate axes $\left(m_{13}=m_{31}=\gamma\right)$, whereas those in the fourth row and fourth column represent linear birefringence along the coordinate axes $\left(m_{34}=-m_{43}=\eta\right)$ and linear birefringence along the bisectors of the coordinate axes $\left(m_{24}=-m_{42}=\nu\right)$.

Also note that the most general $m$ matrix can be written as the sum of two matrices, a symmetric matrix $\mathrm{m}_{s}$ which represents the isotropic and anisotropic absorption properties of the medium and a skew-symmetric matrix $m_{s s}$ which represents the anisotropic refraction properties of the same medium:

$$
\mathrm{m}=\mathrm{m}_{\mathrm{s}}+\mathrm{m}_{s s},
$$

where

$$
\begin{aligned}
\mathrm{m}_{s} & =\left(\begin{array}{cccc}
\alpha & \beta & \gamma & \delta \\
\beta & \alpha & 0 & 0 \\
\gamma & 0 & \alpha & 0 \\
\delta & 0 & 0 & \alpha
\end{array}\right), \\
\mathrm{m}_{s s} & =\left(\begin{array}{cccc}
0 & 0 & 0 & 0 \\
0 & 0 & \mu & \nu \\
0 & -\mu & 0 & \eta \\
0 & -\nu & -\eta & 0
\end{array}\right) .
\end{aligned}
$$

D. The differential matrix $\mathrm{m}$ for a depolarizing medium

We have already seen in Sec. III C that the most general matrix $m$ of a nondepolarizing medium has seven independent real parameters at most and that the elements along the main diagonal are equal, while each pair of off-diagonal elements symmetrically located with respect to the main diagonal are equal or differ only in sign. In the presence of depolarization, we may have more than seven independent elements in the matrix $m$ and the foregoing symmetry properties are broken. The number of independent elements (up to sixteen) and the nature of the $m$ matrix will depend on the physical mechanisms that cause depolarization. The determination of the $m$ matrices that represent media with depolarization is an important separate problem that we will not discuss here. A simple example will, however, be considered in Sec. VI.

\section{LIGHT PROPAGATION IN HOMOGENEOUS NONDEPOLARIZING ANISOTROPIC MEDIA}

For a homogeneous medium $\mathrm{m}$ is constant independent of $z$ and $\mathrm{Eq}$. (11) can be readily integrated to give the
Mueller matrix of a slab of thickness $z$ in terms of the differential matrix $m$ as follows:

$$
\begin{aligned}
\mathbf{M} & =\exp (\mathrm{m} z) \\
& =\mathrm{I}+\mathrm{m} z+\mathrm{m}^{2}\left(z^{2} / 2 !\right)+\mathrm{m}^{3}\left(z^{3} / 3 !\right)+\cdots,
\end{aligned}
$$

where $I$ is the $4 \times 4$ identity matrix as before and the condition $\mathrm{M}(z=0)=\mathrm{I}$ has been used. Therefore to determine $M$ we need to determine the powers of $\mathrm{m}$ and use the exponential series of Eq. (37). To find the powers of $\mathrm{m}$, we write

$$
\mathrm{m}=\mathrm{Tm}_{\mathrm{D}} \mathrm{T}^{-1}
$$

where $T$ is the matrix that transforms $m$ into the diagonal form $\mathrm{m}_{D}$ according to Eq. (38). The diagonal elements of the diagonal matrix

$$
\mathrm{m}_{D}=\left(\begin{array}{cccc}
v_{1} & 0 & 0 & 0 \\
0 & v_{2} & 0 & 0 \\
0 & 0 & v_{3} & 0 \\
0 & 0 & 0 & v_{4}
\end{array}\right)
$$

are the eigenvalues of $\mathrm{m}$ which, in turn, are the roots of the characteristic equation

$$
\operatorname{det}(\mathrm{m}-v \mathrm{I})=0,
$$

and det indicates "the determinant of" and I is the unit matrix. The transfor mation matrix $\mathrm{T}$ can be partitioned into columns

$$
\mathrm{T}=\left[\mathrm{S}_{1} \mathrm{~S}_{2} \mathrm{~S}_{3} \mathrm{~S}_{4}\right] \text {, }
$$

where $S_{i}(i=1, \ldots, 4)$ are the eigenvectors of $\mathrm{m}$ associated with the eigenvalues $v_{i}(i=1, \ldots, 4) . \mathbf{S}_{i}$ are obtained by solving, for each eigenvalue, the set of four homogeneous linear algebraic equations equivalent to the matrix eigenvalue equation

$$
\mathrm{mS}_{i}=v_{i} \mathrm{~S}_{i} \text {. }
$$

The advantage of writing $\mathrm{m}$ in the form of $\mathrm{Eq}$. (38) is that the $n$th power of $m$ is then given by

$$
\mathrm{m}^{n}=\mathrm{Tm}_{D}^{n} \mathrm{~T}^{-1},
$$

as can be readily verified. Raising the diagonal matrix $\mathrm{m}_{D}$ to the $n$th power is simply achieved by raising each and all of its diagonal elements to the same power. If we substitute Eq. (43) into Eq. (37), we obtain

$$
\mathrm{M}=\mathrm{T}\left[\mathrm{I}+\mathrm{m}_{D} z+\mathrm{m}_{D}^{2}\left(z^{2} / 2 !\right)+\cdots\right] \mathrm{T}^{-1},
$$

or

$$
\mathrm{M}=\mathrm{TM}_{D} \mathrm{~T}^{-1},
$$

where

$$
\mathrm{M}_{D}=\exp \left(\mathrm{m}_{D} z\right)=\mathrm{I}+\mathrm{m}_{D} z+\mathrm{m}_{D}^{2}\left(z^{2} / 2 !\right)+\cdots .
$$

Substituting Eq. (39) into Eq. (46), we obtain ${ }^{10}$

$$
\mathbf{M}_{D}=\left(\begin{array}{cccc}
e^{v_{1} z} & 0 & 0 & 0 \\
0 & e^{v_{2} z} & 0 & 0 \\
0 & 0 & e^{v_{3} z} & 0 \\
0 & 0 & 0 & e^{v_{4} z}
\end{array}\right)
$$

Finally, from Eqs. (41), (45), and (47), we can explicit- 
ly construct the Mueller matrix $M$ in terms of the eigenvectors and eigenvalues $\left(S_{i}, v_{i}\right), i=1, \ldots, 4$, of the differential matrix $m$ as follows:

$\mathrm{M}=\left[\mathrm{S}_{1} \mathrm{~S}_{2} \mathrm{~S}_{3} \mathrm{~S}_{4}\right]\left(\begin{array}{cccc}e^{v_{1} z} & 0 & 0 & 0 \\ 0 & e^{v_{2} z} & 0 & 0 \\ 0 & 0 & e^{v_{3} z} & 0 \\ 0 & 0 & 0 & e^{v_{4} z}\end{array}\right)\left[\mathrm{S}_{1} \mathrm{~S}_{2} \mathrm{~S}_{3} \mathrm{~S}_{4}\right]^{-1}$

It should be pointed out that the foregoing general approach need not be applied when the matrix $m$ is simple with most of its elements equal to zero. This is the case when the medium exhibits any one of the optical properties listed in Table I. Take for example the case of circular birefringence. The $m$ matrix that corresponds to this optical property reduces Eq. (3) to the following simple set of differential equations for the Stokes parameters: $d S_{0} / d z=d S_{3} / d z=0, d S_{1} / d z=\mu S_{2}$, and $d S_{2} / d z=-\mu S_{1}$. The first pair of equations yield a constant value for $S_{0}$ and $S_{3}$ upon integration, which indicates the conservation of the total intensity and degree of circular polarization. The second pair is also readily integrable to yield $W=W_{0} \exp (-i \mu z)$, where $W=S_{1}+i S_{2}$ and $W_{0}$ is the value of $W$ at $z=0$. If we construct the polari-

$$
\mathrm{T}=\left(\begin{array}{ccc}
0 & 0 & 1 \\
0 & -\left(\gamma^{2}+\delta^{2}\right)^{1 / 2} / \tau & \beta / \tau \sqrt{2} \\
\delta /\left(\delta^{2}+\gamma^{2}\right)^{1 / 2} & -\beta \gamma / \tau\left(\gamma^{2}+\delta^{2}\right)^{1 / 2} & \gamma / \tau \sqrt{2} \\
-\gamma /\left(\delta^{2}+\gamma^{2}\right)^{1 / 2} & \beta \delta / \tau\left(\gamma^{2}+\delta^{2}\right)^{1 / 2} & \delta / \tau \sqrt{2}
\end{array}\right.
$$

The orthonormality of a set of vectors $S_{i}(i=1, \ldots, 4)$ means that

$$
\mathrm{S}_{i}^{t} \mathrm{~S}_{i}=1, \quad \mathrm{~S}_{i}^{t} \mathrm{~S}_{j}=0, \quad i, j=1, \ldots, 4
$$

where $\mathbf{S}^{t}$ is the transpose of $\mathbf{S}$. The orthogonality of the eigenvectors of $\mathrm{m}$ of Eq. (35) is a direct consequence of the fact that this matrix is real symmetric. ${ }^{11}$ It is important to note that only two of the eigenvectors of $m$ (hence of $\mathbf{M}$ ), namely $\mathbf{S}_{3}$ and $\mathbf{S}_{4}$, represent physical polarization states. Such states are clearly the two possi- zation vector $\mathbf{s}$ [Eqs. (6)], we find that $\mathbf{s}$ generates a right circular cone in $\mathbf{s}$ space with its axis coinciding with the $s_{3}$ coordinate axis.

A. Application to nondepolarizing media with. arbitrary absorptive anisotropy

The $\mathrm{m}$ matrix of a nondepolarizing medium with arbitrary absorptive anisotropy is given by Eq. (35). To solve the eigenvalue equation for this matrix we substitute Eq. (35) into Eq. (40); this gives the following quartic characteristic equation:

$$
(\alpha-v)^{2}\left[(\alpha-v)^{2}-\tau^{2}\right]=0,
$$

where

$$
\tau^{2}=\beta^{2}+\gamma^{2}+\delta^{2},
$$

and $\tau$ represents the net dichroism of the medium. The roots of Eq. (49) are

$$
v_{1}=v_{2}=\alpha, \quad v_{3}=\alpha+\tau, \quad v_{4}=\alpha-\tau .
$$

If we substitute each of the eigenvalues of Eq. (51) into Eq. (42) and solve the resulting set of homogeneous linear algebraic equations, we obtain the orthonormal set of eigenvectors that constitute the columns of the following matrix:

$$
\left.\begin{array}{c}
1 \\
-\beta / \tau \sqrt{2} \\
-\gamma / \tau \sqrt{2} \\
-\delta / \tau \sqrt{2}
\end{array}\right)
$$

ble eigenpolarizations of the medium whose associated eigenvalues, for a slab of thickness $z$, are $e^{v_{3} z}$ and $e^{v_{4} z}$. The other two eigenvectors have a mathematical, rather than a physical, reality only. ${ }^{12}$

The transformation matrix T, Eq. (52), with orthonormal column vectors is unitary and its inverse is its transpose, $\mathrm{T}^{-1}=\mathrm{T}^{t}$. Substituting $\mathrm{T}$ and $\mathrm{T}^{-1}$ and the eigenvalues of Eq. (51) into Eq. (48), we obtain

$$
\mathrm{M}_{z}=e^{\alpha z}\left(\begin{array}{cccc}
\cosh \tau z & \beta_{n} \sinh \tau z & \gamma_{n} \sinh \tau z & \delta_{n} \sinh \tau z \\
\beta_{n} \sinh \tau z & 1+\beta_{n}^{2}(\cosh \tau z-1) & \beta_{n} \gamma_{n}(\cosh \tau z-1) & \beta_{n} \delta_{n}(\cosh \tau z-1) \\
\gamma_{n} \sinh \tau z & \beta_{n} \gamma_{n}(\cosh \tau z-1) & 1+\gamma_{n}^{2}(\cosh \tau z-1) & \gamma_{n} \delta_{n}(\cosh \tau z-1) \\
\delta_{n} \sinh \tau z & \beta_{n} \delta_{n}(\cosh \tau z-1) & \gamma_{n} \delta_{n}(\cosh \tau z-1) & 1+\delta_{n}^{2}(\cosh \tau z-1)
\end{array}\right)
$$

where

$$
\beta_{n}=\beta / \tau, \quad \gamma_{n}=\gamma / \tau, \quad \delta_{n}=\delta / \tau ; \quad \beta_{n}^{2}+\gamma_{n}^{2}+\delta_{n}^{2}=1 .
$$

$\mathrm{M}_{z}$ of Eq. (54) is the Mueller matrix of a slab of thickness $z$ of the medium with arbitrary absorptive anisotropy. In terms of $\mathrm{M}_{z}$, the general solution of Eq. (3) is

$$
\mathbf{S}(z+d)=\mathbf{M}_{z} \mathbf{S}(d)
$$

where $\mathbf{S}(d)$ and $\mathbf{S}(z+d)$ are the Stokes vectors of the light beam at two transverse planes a distance $z$ apart.

As a simple example, consider the propagation of initially unpolarized light through the medium with arbitrary dichroism. Premultiplying the Stokes vector $S_{I}=(1,0$, 0,0 ) by the matrix of Eq. (54), we obtain 


$$
\mathrm{S}(z)=e^{\alpha z}\left(\begin{array}{c}
\cosh \tau z \\
\beta_{n} \sinh \tau z \\
\gamma_{n} \sinh \tau z \\
\delta_{n} \sinh \tau z
\end{array}\right)
$$

The total intensity varies with distance according to $e^{\alpha z} \cosh \tau z$, while the polarization vector $\mathrm{s}$ is given by

$$
\mathbf{s}=\left(\beta_{n}, \gamma_{n}, \delta_{n}\right) \tanh \tau z,
$$

which is fixed in direction ${ }^{13}$ but whose length, the degree of polarization, increases as a hyperbolic-tangent function of the distance traveled. Figure 3 shows the radial trajectory of the point that represents the state of polarization in s space, and also the hyperbolic-tangent growth of the degree of polarization. The propagation of light with an initial polarization other than the unpolarized state can be examined in exactly the same fashion.

B. Application to nondepolarizing media with arbitrary refractive (dispersive) anisotropy

In this case the $m$ matrix is skew symmetric and is given by Eq. (36). The characteristic equation, Eq. (40), for this matrix is

$$
v^{2}\left[v^{2}+\omega^{2}\right]=0,
$$

where

$$
\omega^{2}=\mu^{2}+\nu^{2}+\eta^{2},
$$

and $\omega$ represents the net birefringence of the medium. The roots of Eq. (58) are

$$
v_{1}=v_{2}=0, \quad v_{3}=i \omega, \quad v_{4}=-i \omega .
$$

Only the first pair of eigenvalues, $v_{1}=v_{2}=0$, are physical. If we substitute this doubly degenerate eigenvalue in Eq. (42), the most general solution for the Stokes eigenvector $\mathbf{S}$ is found to be

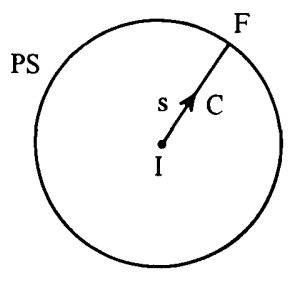

(a)

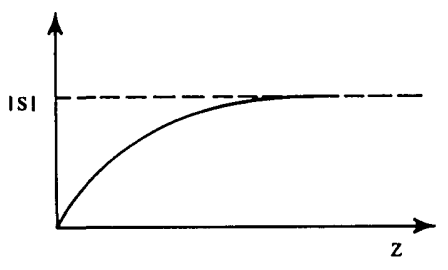

(b)
FIG. 3. (a) The propagation of initially unpolarized light through a nondepolarizing medium with arbitrary absorptive anisotropy is represented by the radial (linear) trajectory $\mathrm{C}$. $I$ and $\mathbf{F}$ represent the initial and final unpolarized and totally polarized states, respectively. F corresponds to the low-absorption eigenpolarization of the medium and is asymptotically approached as the distance of propagation $z$ is increased. (b) The degree of polarization $|\mathbf{s}|$ of initially unpolarized light grows as a hyperbolic-tangent function of the distance $z$ traveled by such light in a nondepolarizing medium with arbitrary absorptive anisotropy.

$$
\mathbf{S}=S_{0}\left(\begin{array}{c}
1 \\
\eta c \\
-\nu c \\
\mu c
\end{array}\right)
$$

which represents infinitely many partially polarized states corresponding to the different values of the arbitrary constant $c .{ }^{14}$ This infinity of eigenvectors, however, can be generated by the linear superposition of two linearly independent Stokes eigenvectors representing a pair of totally polarized orthogonal states:

$$
\mathbf{S}_{1}=\left(\begin{array}{c}
1 \\
\eta_{n} \\
-\nu_{n} \\
\mu_{n}
\end{array}\right), \quad \mathbf{S}_{2}=\left(\begin{array}{c}
1 \\
-\eta_{n} \\
\nu_{n} \\
-\mu_{n}
\end{array}\right),
$$

where

$$
\eta_{n}=\eta / \omega, \quad \nu_{n}=\nu / \omega, \quad \mu_{n}=\mu / \omega ; \quad \eta_{n}^{2}+\nu_{n}^{2}+\mu_{n}^{2}=1 .(63)
$$

Substituting the other pair of eigenvalues, $v_{3}=i \omega, v_{4}$ $=-i \omega$, into Eq. (42), we get the following pair of (only mathematically significant) complex-conjugate eigenvectors ${ }^{12}$ :

$\mathrm{S}_{3}=\left(\begin{array}{c}0 \\ \eta_{n}^{2}-1 \\ -\eta_{n} \nu_{n}-i \mu_{n} \\ \mu_{n} \eta_{n}-i \nu_{n}\end{array}\right), \quad \mathbf{S}_{4}=\left(\begin{array}{c}0 \\ \eta_{n}^{2}-1 \\ -\eta_{n} \nu_{n}+i \mu_{n} \\ \mu_{n} \eta_{n}+i \nu_{n}\end{array}\right)$

The transformation matrix $\mathrm{T}$ that diagonalizes $\mathrm{m}, \mathrm{Eq}$. (38), is constructed from $S_{1}, S_{2}, S_{3}$, and $S_{4}$ as columns:

$$
\mathbf{T}=\left(\begin{array}{cccc}
1 & 1 & 0 & 0 \\
\eta_{n} & -\eta_{n} & \eta_{n}^{2}-1 & \eta_{n}^{2}-1 \\
-\nu_{n} & \nu_{n} & -\eta_{n} \nu_{n}-i \mu_{n} & -\eta_{n} \nu_{n}+i \mu_{n} \\
\mu_{n} & -\mu_{n} & \mu_{n} \eta_{n}-i \nu_{n} & \mu_{n} \eta_{n}+i \nu_{n}
\end{array}\right)
$$

Inverting this matrix, we get

$$
\mathrm{T}^{-1}=\frac{1}{2}\left(\begin{array}{cccc}
1 & \eta_{n} & -\nu_{n} & \mu_{n} \\
1 & -\eta_{n} & \nu_{n} & -\mu_{n} \\
0 & -1 & \frac{-\eta_{n} \nu_{n}+i \mu_{n}}{\mu_{n}^{2}+\nu_{n}^{2}} & \frac{\eta_{n} \mu_{n}+i \nu_{n}}{\mu_{n}^{2}+\nu_{n}^{2}} \\
0 & -1 & \frac{-\eta_{n} \nu_{n}-i \mu_{n}}{\mu_{n}^{2}+\nu_{n}^{2}} & \frac{\eta_{n} \mu_{n}-i \nu_{n}}{\mu_{n}^{2}+\nu_{n}^{2}}
\end{array}\right) .
$$

Finally, to find the Mueller matrix of $\mathrm{M}_{z}$ of a slab of the medium of thickness $z$, we substitute Eqs. (60), (64), and (65) into Eq. (48) and carry out the matrix multiplications; this yields 


$$
\mathrm{M}_{z}=\left(\begin{array}{cccc}
1 & 0 & 0 & 0 \\
0 & \cos \omega z+\eta_{n}^{2}(1-\cos \omega z) & \mu_{n} \sin \omega z-\nu_{n} \eta_{n}(1-\cos \omega z) & \nu_{n} \sin \omega z+\mu_{n} \eta_{n}(1-\cos \omega z) \\
0 & -\mu_{n} \sin \omega z-\nu_{n} \eta_{n}(1-\cos \omega z) & \cos \omega z+\nu_{n}^{2}(1-\cos \omega z) & \eta_{n} \sin \omega z-\mu_{n} \nu_{n}(1-\cos \omega z) \\
0 & -\nu_{n} \sin \omega z+\mu_{n} \eta_{n}(1-\cos \omega z) & -\eta_{n} \sin \omega z-\mu_{n} \nu_{n}(1-\cos \omega z) & \cos \omega z+\mu_{n}^{2}(1-\cos \omega z)
\end{array}\right)
$$

Notice that $\mathbf{M}_{z}$ of Eq. (66) preserves the total intensity (the Stokes parameter $S_{0}$ ) of the beam, as is expected in a transparent medium. On the other hand, the three remaining Stokes parameters $S_{1}, S_{2}$, and $S_{3}$ are transformed among themselves in the course of propagation according to the $3 \times 3$ matrix obtained from $\mathrm{M}_{z}$ by deleting the first row and first column.

As an example, consider the propagation of initially right circularly polarized light through the medium with arbitrary refractive anisotropy. Premultiplying the Stokes vector for such light, $S_{I}=(1,0,0,1)$, by the matrix of Eq. (66), we obtain

$$
\begin{aligned}
& S_{0}=1, \\
& S_{1}=\mu_{n} \eta_{n}(1-\cos \omega z)+\nu_{n} \sin \omega z, \\
& S_{2}=-\mu_{n} \nu_{n}(1-\cos \omega z)+\eta_{n} \sin \omega z, \\
& S_{3}=\mu_{n}^{2}(1-\cos \omega z)+\cos \omega z .
\end{aligned}
$$

The reader can easily verify that the Stokes parameters of Eqs. (67) satisfy the relationship $S_{1}^{2}+S_{2}^{2}+S_{3}^{2}=S_{0}^{2}=1$, indicating the preservation of the degree of polarization, as is expected when light propagates through a nondepolarizing medium. Furthermore, by elimination of $z$ from Eqs. (67), we find that

$$
\eta_{n} S_{1}-\nu_{n} S_{2}+\mu_{n} S_{3}=\mu_{n},
$$

which is the equation of a plane perpendicular to the unit vector $\left(\eta_{n},-\nu_{n}, \mu_{n}\right)$ and which passes through the point $\left(S_{1}, S_{2}, S_{3}\right)=(0,0,1)$. The locus of the state of polarization, as is completely described by the vector $\mathbf{s}$ of $\mathbf{E q}$. (6b), is the circle of intersection of the plane of Eq. (68) with the Poincaré sphere. ${ }^{15}$ The center point of the spherical cab bounded by this circular trajectory represents one of the two eigenpolarizations of the medium (Fig. 4). The reader can further verify that the terminus of $s$ periodically describes the circular trajectory at a uniform rate, as the beam progresses through the medium.

C. Media with combined and arbitrary birefringence and dichroism

The $\mathrm{m}$ matrix of the general nondepolarizing medium with combined arbitrary birefringence and dichroism is

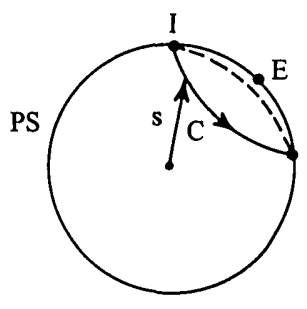

FIG. 4. The propagation of initially right circularly polarized light (I) through a nondepolarizing medium with arbitrary refractive anisotropy is represented by the circle $\mathrm{C}$ on the Poincare sphere (PS) The center point $E$ of the spherical cap bounded by $\mathrm{C}$ represents one of the two orthogonal eigenpolarizations of the medium. given by Eq. (34) as the sum of a symmetric (absorptive) and skew-symmetric (refractive) components. The eigenvalues in this case are the roots of the following quartic equation:

$$
\begin{aligned}
& (\alpha-v)^{4}+(\alpha-v)^{2}\left[\left(\mu^{2}+\nu^{2}+\eta^{2}\right)-\left(\beta^{2}+\gamma^{2}+\delta^{2}\right)\right] \\
& +2[\beta \gamma \nu \eta-\beta \delta \mu \eta+\gamma \delta \mu \nu]=0 .
\end{aligned}
$$

The same steps as have been applied to media with either birefringence or dichroism alone can be used in the present general case. However, finding and inverting the matrix of eigenvectors $\mathrm{T}$, and substituting the results in Eq. (48) to get $M$, involve lengthy and tedious algebra and will be left out.

\section{PROPAGATION OF LIGHT IN INHOMOGENEOUS NONDEPOLARIZING ANISOTROPIC MEDIA}

For a medium which is inhomogeneous along the direction of propagation the matrix $m$ becomes a function of $z$. Analytical solutions for the Stokes-vector differential equation, Eq. (3), become feasible only in a few special cases. However, a general procedure that can be applied in this general case involves the division of the medium into a stack of slabs such that $m$ can be considered sufficiently constant within each slab. This permits the application of the results of Sec. IV to each slab separately, leading to the determination of its Mueller matrix. Multiplying, in proper order, the Mueller matrices of all the slabs accomodated within a given thickness gives the Mueller matrix of the total slab of that thickness.

We consider here the problem of propagation of partially polarized light along the helical axis of a cholesteric or twisted-nematic liquid crystal. The large number of earlier treatments ${ }^{16}$ of this classic problem have been concerned with the propagation of totally polarized light only. ${ }^{17}$

We assume the helically twisted birefringence model. We take the principal axes of the first molecular plane (where the light impinges on the crystal) to be aligned with the $x$ and $y$ axes of an $x y z$ reference Cartesian coordinate system whose $z$ axis is the helical axis of the structure and also the direction of propagation. The $\mathrm{m}$ matrix of the liquid crystal at a distance $z$ is given by

$$
\begin{aligned}
& \mathrm{m}=\mathrm{R}(-\rho z) \mathrm{m}_{0} \mathrm{R}(\rho z), \\
& \rho=2 \pi / p,
\end{aligned}
$$

where $m_{0}$ is the $m$ matrix of the crystal at $z=0$ and $p$ is the pitch of the helical structure. $m_{0}$ is the same as the matrix that represents linear birefringence along the coordinate axes listed in Table I, and $R$ is given by $\mathbf{E q}$. (17). Substitution of these matrices into Eq. (70) gives 


$$
\mathrm{m}=\left(\begin{array}{cccc}
0 & 0 & 0 & 0 \\
0 & 0 & 0 & \eta \sin 2 \rho z \\
0 & 0 & 0 & \eta \cos 2 \rho z \\
0 & -\eta \sin 2 \rho z & -\eta \cos 2 \rho z & 0
\end{array}\right),
$$

where $\eta \lambda / 4 \pi$ is the birefringence of the liquid crystal, Eq. (19). If we substitute $\mathrm{m}$ of Eq. (71) into Eq. (3), we obtain the following set of four linear first-order differential equations in the Stokes parameters:

$$
\begin{aligned}
& d S_{0} / d z=0, \\
& d S_{1} / d z=(\eta \sin 2 \rho z) S_{3}, \\
& d S_{2} / d z=(\eta \cos 2 \rho z) S_{3}, \\
& d S_{3} / d z=(-\eta \sin 2 \rho z) S_{1}+(-\eta \cos 2 \rho z) S_{2} .
\end{aligned}
$$

Equation (72a) is readily integrated to give $S_{0}=$ constant, meaning that the total intensity is conserved, as would be expected in a transparent medium. Differentiating Eq. (72d) once and making use of Eqs. (72b) and (72c) we obtain

$$
\left(d^{2} S_{3} / d z^{2}\right)+\eta^{2} S_{3}=2 \eta \rho\left(-S_{1} \cos 2 \rho z+S_{2} \sin 2 \rho z\right) .
$$

Eliminating $S_{2}$ between Eqs. (72d) and (73), we get

$$
\begin{aligned}
- & S_{1}=(\sin 2 \rho z / \eta)\left(d S_{3} / d z\right)+(\cos 2 \rho z / 2 \eta \rho)\left(d^{2} S_{3} / d z^{2}\right) \\
& +(\eta \cos 2 \rho z / 2 \rho) S_{3} .
\end{aligned}
$$

Next, we differentiate Eq. (74) once and substitute $d S_{1} /$ $d z$ from Eq. (72b); this give a third-order linear differential equation with constant coefficients in $S_{3}$ only:

$$
\left(d S_{3}^{3} / d z^{3}\right)+\left(\eta^{2}+4 \rho^{2}\right)\left(d S_{3} / d z\right)=0 .
$$

Equation (75) is readily integrable by standard techniques ${ }^{18}$ and its general solution is given by

$$
\begin{aligned}
& S_{3}=c_{1}+c_{2} \sin \left(\zeta z+c_{3}\right), \\
& \zeta^{2}=\eta^{2}+4 \rho^{2},
\end{aligned}
$$

where $c_{1}, c_{2}$, and $c_{3}$ are arbitrary constants of integration. If we substitute $S_{3}$ of Eq. (76) into Eqs. (72b, c) and integrate the resulting equations once, we get

$$
\begin{aligned}
S_{1}= & c_{4}+\left(-\eta c_{1} / 2 \rho\right) \cos 2 \rho z \\
& +\left[\eta c_{2} / 2(2 \rho-\zeta)\right] \sin \left[(2 \rho-\zeta) z-c_{3}\right] \\
& +\left[-\eta c_{2} / 2(2 \rho+\zeta)\right] \sin \left[(2 \rho+\zeta) z+c_{3}\right], \\
S_{2}= & c_{5}+\left(\eta c_{1} / 2 \rho\right) \sin 2 \rho z \\
& +\left[-\eta c_{2} / 2(2 \rho+\zeta)\right] \cos \left[(2 \rho+\zeta) z+c_{3}\right] \\
& +\left[\eta c_{2} / 2(2 \rho-\zeta)\right] \cos \left[(2 \rho-\zeta) z-c_{3}\right],
\end{aligned}
$$

where $c_{4}$ and $c_{5}$ are additional arbitrary constants of integration. Equations (72b, c, d) should lead to three arbitrary constants at most, so that the five constants of integration $c_{1}, \ldots, c_{5}$ should be reducible to three only. If we substitute Eqs. (76), (77), and (78) into Eq. (72d), the result can be written as the sum of different Fourier components of different frequencies equal to zero. This means that the amplitude coefficients in this series must vanish identically, from which we have found that $c_{4}$ and $c_{5}$ are equal to zero.

To obtain the three remaining arbitrary constants $c_{1}$, $c_{2}$, and $c_{3}$, we use the initial condition that at $z=0$ the Stokes parameters $S_{1}, S_{2}$, and $S_{3}$ are equal to their initial values $S_{10}, S_{20}$, and $S_{30}$, respectively. This gives

$$
\begin{aligned}
& c_{1}=\left(4 \rho^{2} / \zeta^{2}\right) S_{30}+\left(-2 \eta \rho / \zeta^{2}\right) S_{10}, \\
& c_{2} \sin c_{3}=\left(\eta^{2} / \zeta^{2}\right) S_{30}+\left(2 \eta \rho / \zeta^{2}\right) S_{10}, \\
& c_{2} \cos c_{3}=(-\eta / \zeta) S_{20} .
\end{aligned}
$$

The final solution for the Stokes vector of light propagating along the helical axis of the cholesteric liquid crystal can be put in the form of Eq. (8), where $\mathbf{M}_{\boldsymbol{z}}$ is the Mueller matrix of a slab of thickness $z$ (a typical sandwich structure) whose elements are explicitly given by

$$
\begin{aligned}
M_{11}= & 1, \quad M_{12}=M_{13}=M_{14}=M_{21}=M_{31}=M_{41}=0 ; \\
M_{22}= & \left(\rho \eta^{2} / \zeta^{2}\right)\left(\frac{\cos 2 \rho z}{\rho}-\frac{\cos (2 \rho+\zeta) z}{2 \rho+\zeta}-\frac{\cos (2 \rho-\zeta) z}{2 \rho-\zeta}\right), \\
M_{23}= & \left(\eta^{2} / 2 \zeta\right)\left(\frac{\sin (2 \rho+\zeta) z}{2 \rho+\zeta}-\frac{\sin (2 \rho-\zeta) z}{2 \rho-\zeta}\right), \\
M_{24}= & \left(-\eta / 2 \zeta^{2}\right) \\
& \times\left(4 \rho \cos 2 \rho z+\eta^{2} \frac{\cos (2 \rho+\zeta) z}{2 \rho+\zeta}+\eta^{2} \frac{\cos (2 \rho-\zeta) z}{2 \rho-\zeta}\right), \\
M_{32}= & \left(\rho \eta^{2} / \zeta^{2}\right)\left(-\frac{\sin 2 \rho z}{\rho}+\frac{\sin (2 \rho+\zeta) z}{2 \rho+\zeta}+\frac{\sin (2 \rho-\zeta) z}{2 \rho-\zeta}\right), \\
M_{33}= & \left(\eta^{2} / 2 \zeta\right)\left(\frac{\cos (2 \rho+\zeta) z}{2 \rho+\zeta}-\frac{\cos (2 \rho-\zeta) z}{2 \rho-\zeta}\right), \\
M_{34}= & \left(\eta / 2 \zeta^{2}\right)\left(4 \rho \sin 2 \rho z+\eta^{2} \frac{\sin (2 \rho+\zeta) z}{2 \rho+\zeta}\right. \\
& \left.+\eta^{2} \frac{\sin (2 \rho-\zeta) z}{2 \rho-\zeta}\right),
\end{aligned}
$$

$M_{42}=\left(-2 \rho \eta / \zeta^{2}\right)(1-\cos \zeta z), \quad M_{43}=(-\eta / \zeta) \sin \zeta z$,

$M_{44}=\left(4 \rho^{2}+\eta^{2} \cos \zeta z\right) / \zeta^{2}$.

Equations (80) can be used to derive many of the specific optical properties of the cholesteric liquid crystal previously deduced using other solution techniques, as the reader can readily prove. ${ }^{19}$

\section{PROPAGATION OF LIGHT IN DEPOLARIZING MEDIA}

We have seen in Sec. III that the $\mathrm{m}$ matrix of a nondepolarizing medium has the following properties: (1) all diagonal elements are equal, and (2) off-diagonal elements symmetrically located with respect to the main diagonal are either equal or differ only in sign. These symmetry properties of the $m$ matrix lead to seven independent optical parameters that control the intensity and the state of polarization of light propagating through such a medium. Breaking any of these symmetry conditions produces a matrix $\mathrm{m}$ that must correspond to a depolarization phenomenon, or else a matrix that is entirely nonphysical. Furthermore, up to 16 independent 
parameters (the 16 elements of the $4 \times 4$ matrix $\mathrm{m}$ ) may be necessary to describe the evolution of the Stokes vector of light in the medium. It is outside the scope of this paper to attempt to derive the $m$ matrices that may correspond to the many different possible physical processes that can cause depolarization. Instead, and for the purpose of illustration, let us assume an $\mathrm{m}$ matrix with all of its elements equal to zero except $m_{44}$ :

$$
m=\left(\begin{array}{cccc}
0 & 0 & 0 & 0 \\
0 & 0 & 0 & 0 \\
0 & 0 & 0 & 0 \\
0 & 0 & 0 & -\sigma
\end{array}\right),
$$

where $\sigma$ is a positive constant (i.e., the medium is homogeneous). This matrix may represent a depolarization process because it breaks the rule of equality of the diagonal elements which the matrix $m$ of a nondepolarizing medium must satisfy. It we substitute Eq. (81) into Eq. (3), we obtain

$$
\begin{aligned}
& d S_{0} / d z=d S_{1} / d z=d S_{2} / d z=0, \\
& d S_{3} / d z=-\sigma S_{3} .
\end{aligned}
$$

Integrating Eqs. (82a), we readily find that $S_{0}, S_{1}$, and $S_{2}$ are constant independent of $z$, indicating the conservation of the total intensity, the horizontal preference, and the $+45^{\circ}$ preference, respectively. On the other hand, the integral of Eq. (82b) yields

$$
S_{3}=S_{30} e^{-\sigma z} \text {. }
$$

The exponential decay of $S_{3}$ as a function of distance along the direction of propagation indicates a depolarization mechanism that produces gradual equalization of the proportions of the total intensity in the right and left circularly polarized states. Thus incident light polarized in any initial state eventually becomes (partially) linearly polarized after a sufficient distance of propagation. The trajectory of the point that represents the state of polarization in $\mathbf{s}$ space, Sec. $I$, is a vertical line joining the point of initial polarization I and its projection $\mathrm{F}$ in the equatorial $\left(s_{1}, s_{2}\right)$ plane which represents the final state of partial linear polarization (Fig, 5).

Clearly, if the $\mathrm{m}$ matrix of a given depolarizing medi$\mathrm{um}$ is determined, the evolution of the Stokes vector of a light beam propagating in such a medium can be derived using the same techniques as has been discussed for nondepolarizing media (Secs. IV and V).

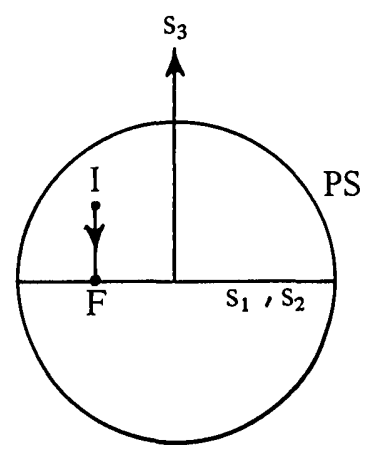

FIG. 5. The propagation of light through a depolarizing medium with the differential matrix given by Eq. (81) is represented by a vertical straight line (parallel to the $s_{3}$ axis) through the point I which represents an arbitrary initial state. The point $F$ which represents the final state of partial linear polarization is in the equatorial $\left(s_{1} s_{2}\right)$ plane.

\section{CONCLUDING REMARKS}

We have introduced a differential matrix calculus to describe the continuous propagation of partially polarized light through linear anisotropic media that may exhibit depolarization. The calculus is the $4 \times 4$ analog, in the Stokes vector-Mueller matrix formalism, of Jones $2 \times 2$ differential calculus suited for the case of propagation of totally polarized light in nondepolarizing anisotropic media.

Our real matrix calculus is distinct from the $4 \times 4$ complex matrix calculus of Berreman and other $\mathrm{s}^{20}$ which also describes light propagation in stratified anisotropic structures. The most fundamental difference is that the wave vector in our calculus is the phenomenological Stokes vector, whereas the wave vector in Berreman's calculus consists of the transverse components of the electric and magnetic field vectors. Another important difference is that our calculus can handle depolarization effects, whereas that of Berreman is not designed for this purpose. For a nondepolarizing medium it should be possible to relate our differential matrix $m$ to Berreman's differential matrix $\Delta$ (hence, the matrix $M$ of the dielectric, permeability, and optical rotation tensors). In this regard, it should be noted that, in the last of his series of papers on his calculus, ${ }^{21}$ Jones determined the relationship between his differential matrix $\mathrm{N}$ and the dielectric and gyration tensors of the medium. Because $\mathrm{m}$ has been related to $\mathrm{N}$ (see Sec. III C), the determination of $m$ in terms of the basic optical properties of the medium (i.e., the dielectric and gyration tensors) is automatically accomplished.

Finally, although we have referred to light waves in particular throughout this paper, the results are obviously applicable to the propagation of other electromagnetic radiation through anisotropic media.

${ }^{1}$ R. C. Jones, "A New Calculus for the Treatment of Optical Systems. VII, Properties of the N-Matrices," J. Opt. Soc. Am. 38, 671-685 (1948).

${ }^{2} \mathrm{An}$ alternative related description is that based on the complex polarization variable $\chi$ (equal to the ratio of the complex amplitudes of two orthogonal components of $\mathrm{E}$ ) which obeys Riccati's differential equation. See R. M. A. Azzam and N. M. Bashara, "Simplified Approach to the Propagation of Polarized Light in Anisotropic Media-Application to Liquid Crystals," J. Opt. Soc. Am. 62, 1252-1257 (1972).

${ }^{3}$ See, for example, R. M. A. Azzam and N. M. Bashara, Ellipsometry and Polarized Light (North-Holland, Amsterdam, 1977).

${ }^{4}$ For simplicity, we drop the argument or subscript $z$, which indicates $z$ dependence, from $S$ and $m$. Equations (3) and (4) are the $4 \times 4$-matrix analogs of Eqs. $(2.10)$ and $(2.2)$, respectively, in Ref. 1.

${ }^{5}$ R. M. A. Azzam, B. E. Merrill, and N. M. Bashara, "Trajectories Describing the Evolution of Polarized Light in Homogeneous Anisotropic Media and Liquid Crystals," Appl. Opt. 12,764-771 (1973); B. E. Mexrill, R. M. A. Azzam, and N. M. Bashara, "Numerical Solution for the Evolution of the Ellipse of Polarization in Inhomogeneous Anisotropic Media," J. Opt. Soc. Am. 64, 731-733 (1974).

${ }^{6}$ See the $2 \times 2$ Jones-matrix analogs of these equations in Ref. 1 [Eqs. (2.6), (2.7), (2.17), and (2.13)].

${ }^{7}$ The inverse of $M_{z}$ is derived from $M_{z}$ by changing the sign of 
$z$ and the differentiation is elementary.

${ }^{8} d_{+}^{i}$ represents the is otropic refractive properties of the medium.

${ }^{9}$ It can be easily verified that this matrix is the $4 \times 4$ equivalent of the $2 \times 2$ Jones matrix $N$ obtained by adding the eight differential Jones matrices listed in Table $\mathrm{I}$, in accordance with the transformation specified by Eqs. (26) and (29). This provides a check on the latter transformation.

${ }^{10}$ From Eqs. (45) and (47) it can be concluded that for a homogeneous medium (1) the eigenvectors of the intensive differential matrix $m$ and the extensive Mueller matrix $M$ are the same, and (2) an eigenvalue $v_{M}$ of $\mathrm{M}$ is related to the corresponding eigenvalue $v_{m}$ of $\mathrm{m}$ by $v_{M}=\exp \left(v_{m} z\right)$. In Ref. 1 , Jones provides a different proof of the similar relationships between the eigenvectors and eigenvalues of his intensive and extensive $2 \times 2$ matrices.

${ }^{11}$ See, for example, C. R. Wylie, Jr., Advanced Engineering Mathematics (McGraw-Hill, New York, 1966), p. 487.

${ }^{12}$ This is probably the first time that the Mueller-matrix eigenvalue problem has been considered and the significance of its eigenvectors and eigenvalues examined.

${ }^{13}$ With direction cosines $\left(\beta_{n}, \gamma_{n}, \delta_{n}\right)$.

${ }^{14}$ Because the degree of polarization cannot exceed one, $c$ is limited to the range $-1 / \omega \leq c \leq 1 / \omega$. Values of $c$ outside this range yield nonphysical, but mathematically acceptable, eigenvectors.

${ }^{15}$ Notice that because $S_{0}=1, S_{i}=s_{i}(i=1,2,3)$.

${ }^{16}$ See, for example, Ref. 2 and the references cited therein.
${ }^{17}$ The results of previous treatments can be used to account for the propagation of partially polarized light only by decomposing such light into its totally polarized and unpolarized components. The present development is direct in its handling of partial polarization and, more importantly, is capable of analyzing depolarization effects as may be caused, for example, by small random perturbations in the molecular ordering of the liquid crystal.

${ }^{18}$ See Ref. 11, Chap. 2 .

${ }^{19}$ It is interesting to note that, for any initial polarization, the Stokes parameters $S_{1}$ and $S_{2}$ have three spatial-frequency components: one at double the spatial frequency of the helical structure $(2 \rho)$ and the other two $[(2 \rho+\zeta)$ and $(2 \rho-\zeta)]$ are upand down-shifted from this (center) frequency by the same

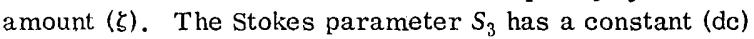
component and one spatial-frequency component $(\zeta)$. If we derive the ellipticity and azimuth of the polarization ellipse of the wave from the Stokes parameters, we will find that they have the same behavior as a function of distance as has been pointed out in Ref. 2.

${ }^{20} \mathrm{D}$. W. Berreman, "Optics in Stratified Anisotropic Media: $4 \times 4$-Matrix Formulation," J. Opt. Soc. Am. 62, 502-510 (1972) and references therein.

${ }^{21} \mathrm{R}$. C. Jones, "New Calculus for the Treatment of Optical Systems. VIII. Electromagnetic Theory," J. Opt. Soc. Am. $46,126-131(1956)$.

\title{
Luminous efficiency functions determined by successive brightness matching
}

\author{
Mitsuo Ikeda and Hiroaki Shimozono \\ Department of Information Processing, Graduate School, Tokyo Institute of Technology, Nagatsuta, Midori-ku, Yokohama 227, \\ Japan
}

(Received 22 May 1978)

The alternating frequency in flicker photometry is normally high enough to go beyond the critical fusion frequency, thus avoiding the interference of color alternation. If, however, the alternating frequency is purposefully lowered, we have new method, a modified direct heterochromatic matching technique, which we have called successive brightness matching. A luminous efficiency function was obtained with this method by an equal brightness criterion at the alternating frequency of $0.5 \mathrm{~Hz}$. The function showed a favorable agreement with the normal direct brightness matching, as we expected. With a similar low frequency, $2 \mathrm{~Hz}$, another luminous efficiency was obtained according to the newly introduced criterion, the minimum-flicker perception, at the transition point of one light to another. The function agreed perfectly with flicker photometry, implying that the criterion detected only the activation in the achromatic channel proposed in the opponent-colors theory. At increasing the alternating frequency successive brightness matching converges to the normal flicker photometry, which was confirmed by the change in luminous efficiency. The transient frequency was about 4 or $6 \mathrm{~Hz}$, which indicated the cutoff frequency of the chromatic channel of the visual system.

\section{INTRODUCTION}

The interest in the luminous efficiency function or the human spectral sensitivity to brightness has recently been revived, ${ }^{1-4}$ probably due to two main reasons. One is simply a need, particularly in industry, to have correct information about the function. Current developments of various light sources such as lasers and LED initiated a common use of monochromatic light in laboratories and in every day life. The brightness specification for these light sources critically depends upon the shape of the luminous efficiency function, and it is quite natural to ask for the establishment of the function to assure a good agreement with the subjective brightness judged directly for these sources. Another reason stems from the immense increase of knowledge about the mechanisms of visual information. Along with this increase, a desire for clearer light on the mechanism of brightness sensation has arisen. This is one of the fundamental properties of the visual system.

The present paper will offer some information about heterochromatic flicker photometry which is often used when 